\title{
Hyperpigmentation following Treatment of Frontal Fibrosing Alopecia
}

\author{
Irma Margarita Pérez-Rodríguez Martha Elena García-Melendez \\ Kristian Eichelmann Osvaldo Vázquez-Martínez Jorge Ocampo- \\ Candiani
}

Department of Dermatology, Hospital Universitario 'Dr. José Eleuterio González', Universidad Autónoma de Nuevo León, Monterrey, Mexico

\section{Key Words}

Hyperpigmentation · Frontal fibrosing alopecia · Scarring alopecia

\begin{abstract}
Introduction: Frontal fibrosing alopecia (FFA) is a scarring alopecia characterized by progressive recession of the frontotemporal hairline. Current treatment is aimed at stopping progression, and the combination of dutasteride and pimecrolimus is the most effective therapy. Side effects associated with dutasteride are erectile dysfunction as well as breast tenderness and enlargement, while pimecrolimus produces a burning sensation. Case Report: We present a 57-year-old postmenopausal female with a 3-year history of a scarring alopecic plaque in her frontotemporal region. Biopsy confirmed the diagnosis of FFA, and she was started on dutasteride $0.5 \mathrm{mg}$ p.o. q.d., and later, topical pimecrolimus $1 \%$ b.i.d. was added. Eight months after initiating treatment, she showed hyperpigmentation on her metacarpophalangeal and interphalangeal joints, as well as on the cheeks and on the chin; dutasteride and pimecrolimus were discontinued. After 5 months of follow-up, her hyperpigmentation improved by $80 \%$ only by using photoprotection. Conclusion: Because of the variable clinical course of FFA, treatment is focused on halting its progression. Several therapeutic agents have been evaluated and the combination of dutasteride and pimecrolimus has shown a high response rate. There is no reported evidence of hyperpigmentation associated with this combination.


Pérez-Rodríguez et al.: Hyperpigmentation following Treatment of Frontal Fibrosing Alopecia

\section{Introduction}

Frontal fibrosing alopecia (FFA) is a scarring alopecia that affects postmenopausal women and is considered a clinical variant of lichen planopilaris. Classic symptoms include erythema, follicular hyperkeratosis and progressive recession of the frontotemporal hairline [1]. Currently, there is no treatment of choice and because of the scarring nature, the aim is to stop progression. Studies have shown that the combination of dutasteride and pimecrolimus is by far the most effective therapy for this condition, and even hair regrowth has been reported $[2,3]$.

\section{Case Report}

We present a 57-year-old postmenopausal female with a 3-year history of scarring alopecic plaques on both her frontotemporal regions, which she developed after an upper third face lift with aptos threads. Dermoscopy showed an absence of follicular openings, perifollicular scaling and erythema. Skin biopsy revealed the presence of a perifollicular lymphocytic infiltrate and lamellar fibrosis, a decrease of hair follicles and apoptotic cells in the external root sheath, therefore confirming the diagnosis of FFA (fig. 1).

Treatment was started with dutasteride $0.5 \mathrm{mg}$ p.o. q.d. At the fifth month of follow-up, we added topical pimecrolimus $1 \%$ b.i.d. At this point, the patient presented a $40 \%$ improvement in erythema and even showed slight hair regrowth.

Eight months after the treatment was started, the patient presented hyperchromic macules in the metacarpophalangeal and interphalangeal joints of both hands as well as on the cheeks and chin (fig. 2); she denied the use of other concomitant treatments. A skin biopsy was performed, revealing basal layer membrane hyperpigmentation.

Treatment was stopped and the use of photoprotection was encouraged. Five months after the treatment was discontinued, the patient showed an $80 \%$ improvement of her hyperpigmented lesions (fig. 3).

\section{Discussion}

FFA was described in 1994 by Kossard in postmenopausal women, but it can also affect premenopausal women and males. It is considered a clinical variant of lichen planopilaris; histologically, both entities show a lymphocytic infiltrate and fibrosis between the follicular epithelium and the dermis, involving the upper mid-portion of the follicle, but FFA shows less inflammatory infiltrate than lichen planopilaris [4]. The pathogenesis is unknown, but a T-cell-mediated inflammatory reaction with the destruction of stem cells and hormonal changes as well as increased levels of androgens are thought to play an important role [5].

FFA has a variable clinical course and the treatment is focused on stopping progression. Several therapeutic agents have been evaluated and topical and systemic corticosteroids have been the most commonly used agents; with a high risk of relapse upon discontinuation [1], other treatments have been studied, e.g. antimalarials, mycophenolate mofetil, doxycycline, and topical minoxidil with variable results [2].

Treatments with $5 \alpha$-reductase inhibitors have shown good results supporting the role of androgens in the pathogenesis of FFA [6]. Dutasteride is a $5 \alpha$-reductase inhibitor, it is 3 times more potent than finasteride at inhibiting type II enzyme and 100 times more potent at inhibiting type I enzyme, making it a leading option in treating FFA. 
Pérez-Rodríguez et al.: Hyperpigmentation following Treatment of Frontal Fibrosing Alopecia

Pimecrolimus, a topical calcineurin inhibitor that blocks T-cell activation and cytokine production, has been used effectively in combination with dutasteride [3].

Reported side effects associated with dutasteride and finasteride use are erectile dysfunction as well as breast tenderness and enlargement [7, 8]. For finasteride, there are reports of cutaneous vasculitis, drug-related folliculitis, erythema annulare centrifugum, solitary fixed drug eruption, urticarial rash and acute generalized exanthematous pustulosis; the last one occurred 3 months after it was initiated [9]. For pimecrolimus, the reported adverse effects are a burning sensation and pruritus [8], and there is one report of induced lentiginosis on the application site of this drug [10]; however, in this case, the hyperpigmentation did not appear at the sites of application of pimecrolimus. There is no reported evidence of hyperpigmentation associated with this combination or with the use of dutasteride.

Another described cause of hyperpigmentation in the presence of alopecia is the Cronkhite-Canada syndrome. It is a rare syndrome involving the skin and the gastrointestinal tract, showing hyperpigmentation, alopecia, onychodystrophy, diarrhea, hamartomatous polyposis and weight loss $[11,12]$. An autoimmune mechanism may be involved, but the etiopathogenesis remains unknown [12].

Initial symptoms are diarrhea and dysgeusia, and the dermatologic triad occurs later [12]. The alopecia in these patients is a non-scarring alopecia, with telogen hair follicles without miniaturization or atrophy of the hair follicle and absence of inflammation [13], which can start in patches and then become total; the hyperpigmentation is observed diffusely in the upper extremities. It shows onychodystrophy which includes a thinning of the nail plate, onycholysis and onychomadesis [11].

In our patient's case, this diagnosis is discarded because she does not have gastrointestinal symptoms, she has a scarring alopecia, and the hyperpigmentation occurred mainly on her face.

\section{Conclusion}

Because of the variable clinical course of FFA, treatment is focused on halting its progression. Several therapeutic agents have been evaluated; the combination of dutasteride and pimecrolimus has shown a high response rate. This case is of particular interest because the patient developed hyperpigmentation following the use of dutasteride and a remission after its discontinuation. There is still no clear evidence that points to dutasteride as the sole precipitating agent; therefore, more studies are required to further evaluate this association.

\section{Disclosure Statement}

The authors declare no conflict of interest.

\section{References}

1 Smidarle D, Seidl M, Castilhos R: Frontal fibrosing alopecia - a case report. An Bras Dermatol 2010;85:879882.

2 Rácz E, Gho C, Moorman PW, Noordhoek Hegt V, Neumann HAM: Treatment of frontal fibrosing alopecia and lichen planopilaris: a systematic review. J Eur Acad Dermatol Venereol 2013, DOI: 10.1111/jdv.12139.

-3 Katoulis A, Georgala S, Bozi E, Papadavid E, Kalogeromitros D, Stavrianeas N: Frontal fibrosing alopecia: treatment with oral dutasteride and topical pimecrolimus. J Eur Acad Dermatol Venereol 2009;23:570-620. 


\begin{tabular}{l|l}
\hline \multicolumn{2}{l}{ Case Rep Dermatol 2013;5:357-362 } \\
\hline DOI: $10.1159 / 000357022$ & $\begin{array}{l}\text { C 2014 S. Karger AG, Basel } \\
\text { www.karger.com/cde }\end{array}$ \\
\hline
\end{tabular}

Pérez-Rodríguez et al:: Hyperpigmentation following Treatment of Frontal Fibrosing Alopecia

4 Poblet E, Jiménez F, Pascual A, Piqué E: Frontal fibrosing alopecia versus lichen planopilaris: a clinicopathological study. Int J Dermatol 2006;45:375-380.

5 Dawn G, Holmes SC, Moffat D, Munro CS: Post-menopausal frontal fibrosing alopecia. Clin Exp Dermatol 2003;28:43-45.

6 Tosti A, Piraccini BM, Iorizzo M, Misciali C: Frontal fibrosing alopecia in postmenopausal women. J Am Acad Dermatol 2005;52:55-60.

$\rightarrow 7$ Kaplan SA, Chung DE, Lee RK, Scofield S, Te AE: A 5-year retrospective analysis of 5a-reductase inhibitors in men with benign prostatic hyperplasia: finasteride has comparable urinary symptom efficacy and prostate volume reduction, but less sexual side effects and breast complications than dutasteride. Int J Clin Pract 2012;66:1052-1055.

8 Wolverton SE: Comprehensive Dermatologic Drug Therapy. Philadelphia, Saunders Elsevier, 2013.

-9 Tresch S, et al: T cell-mediated acute localized exanthematous pustulosis caused by finasteride. J Allergy Clin Immunol 2012;129:589-594.

10 Castelo L, et al: Induced lentiginosis with use of topical calcineurin inhibitors. Arch Dermatol 2012;148:766-767.

11 Ho V, Banney L, Falhammar H: Hyperpigmentation, nail dystrophy and alopecia with generalised intestinal polyposis: Cronkhite-Canada syndrome. Australas J Dermatol 2008;49:223-225.

12 Sweetser S, Boardman L: Cronkhite-Canada syndrome: an acquired condition of gastrointestinal polyposis and dermatologic abnormalities. Gastroenterol Hepatol 2012;8:201-203.

-13 Watanabe-Okada E, Inazumi T, Matsukawa H, Ohyama M: Histopathological insights into hair loss in Cronkhite-Canada syndrome: diffuse anagen-telogen conversion precedes clinical hair loss progression. Australas J Dermatol 2013, DOI: 10.1111/ajd.12068.
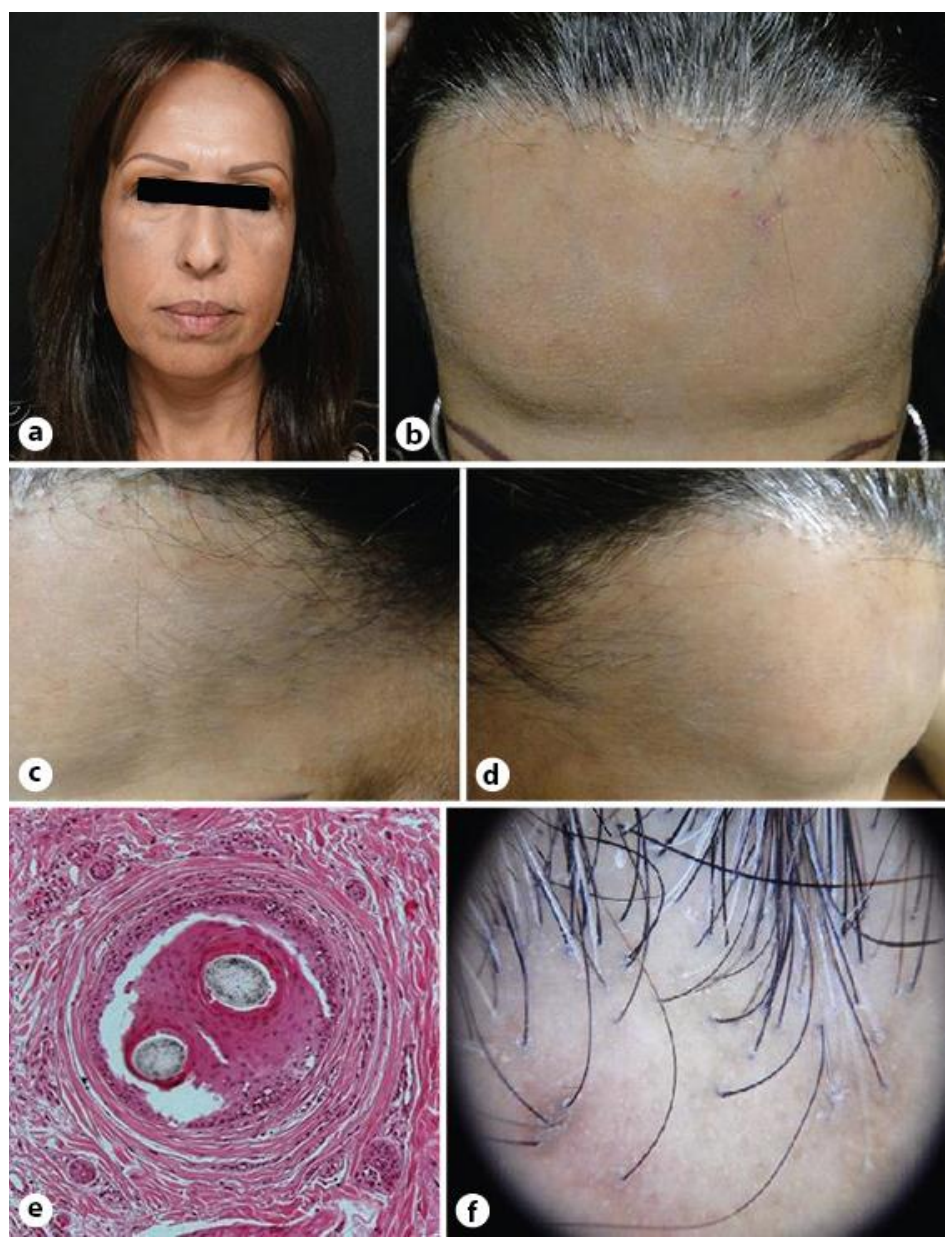

Fig. 1. a-d Female patient with frontotemporal hairline recession. e Histologic image showing lamellar fibrosis. $\mathbf{f}$ Dermoscopic image showing erythema, absence of follicular openings and perifollicular scaling. 


\section{Case Reports in Dermatology}

\begin{tabular}{l|l}
\hline Case Rep Dermatol 2013;5:357-362 & \\
\hline DOI: $10.1159 / 000357022$ & $\begin{array}{l}\text { @ 2014 S. Karger AG, Basel } \\
\text { www.karger.com/cde }\end{array}$ \\
\hline
\end{tabular}

Pérez-Rodríguez et al.: Hyperpigmentation following Treatment of Frontal Fibrosing Alopecia
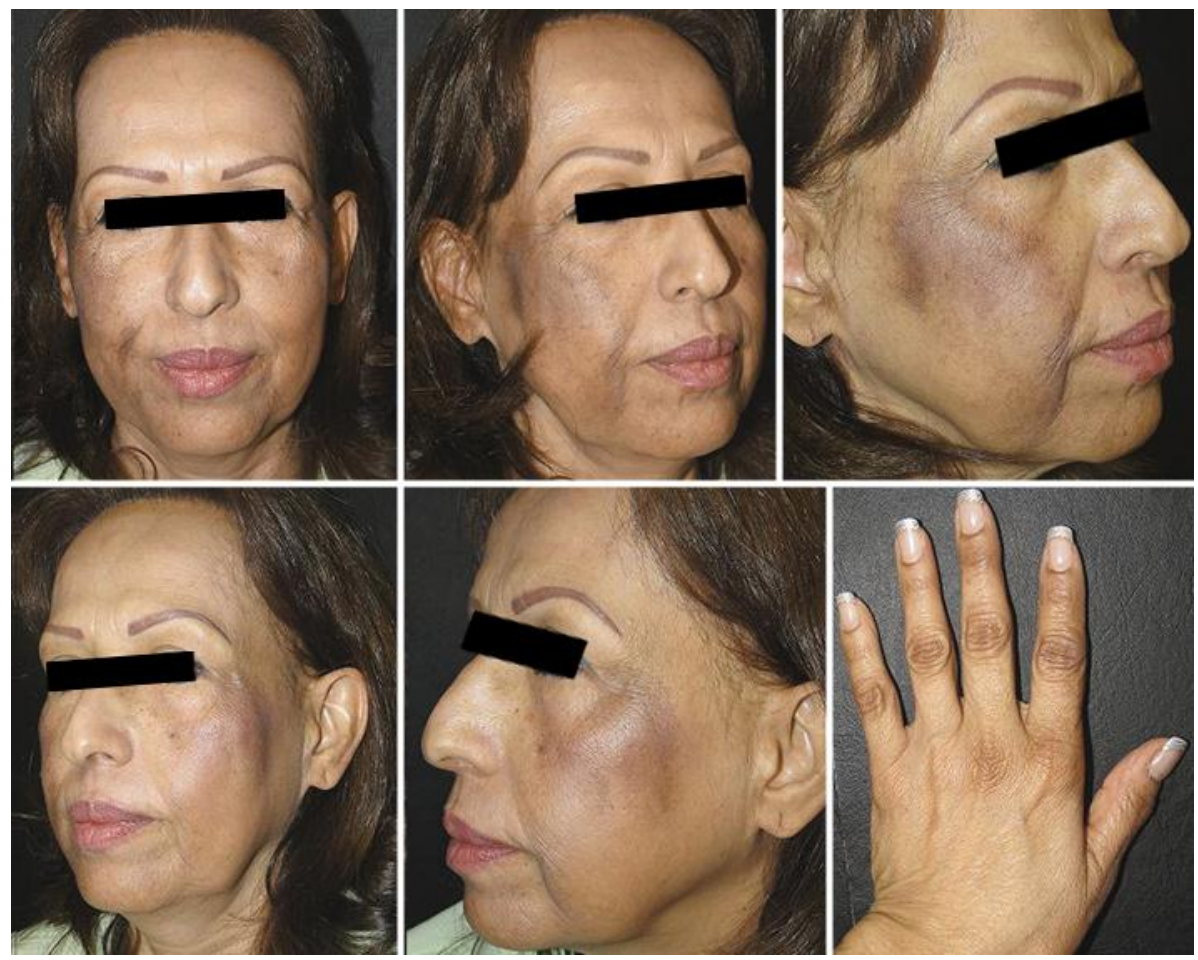

Fig. 2. Eight-month follow-up; the patient presents hyperchromic macules in metacarpophalangeal and interphalangeal joints, cheeks and chin. 


\section{Case Reports in Dermatology}

Case Rep Dermatol 2013;5:357-362

DOI: 10.1159/000357022

Pérez-Rodríguez et al.: Hyperpigmentation following Treatment of Frontal Fibrosing Alopecia
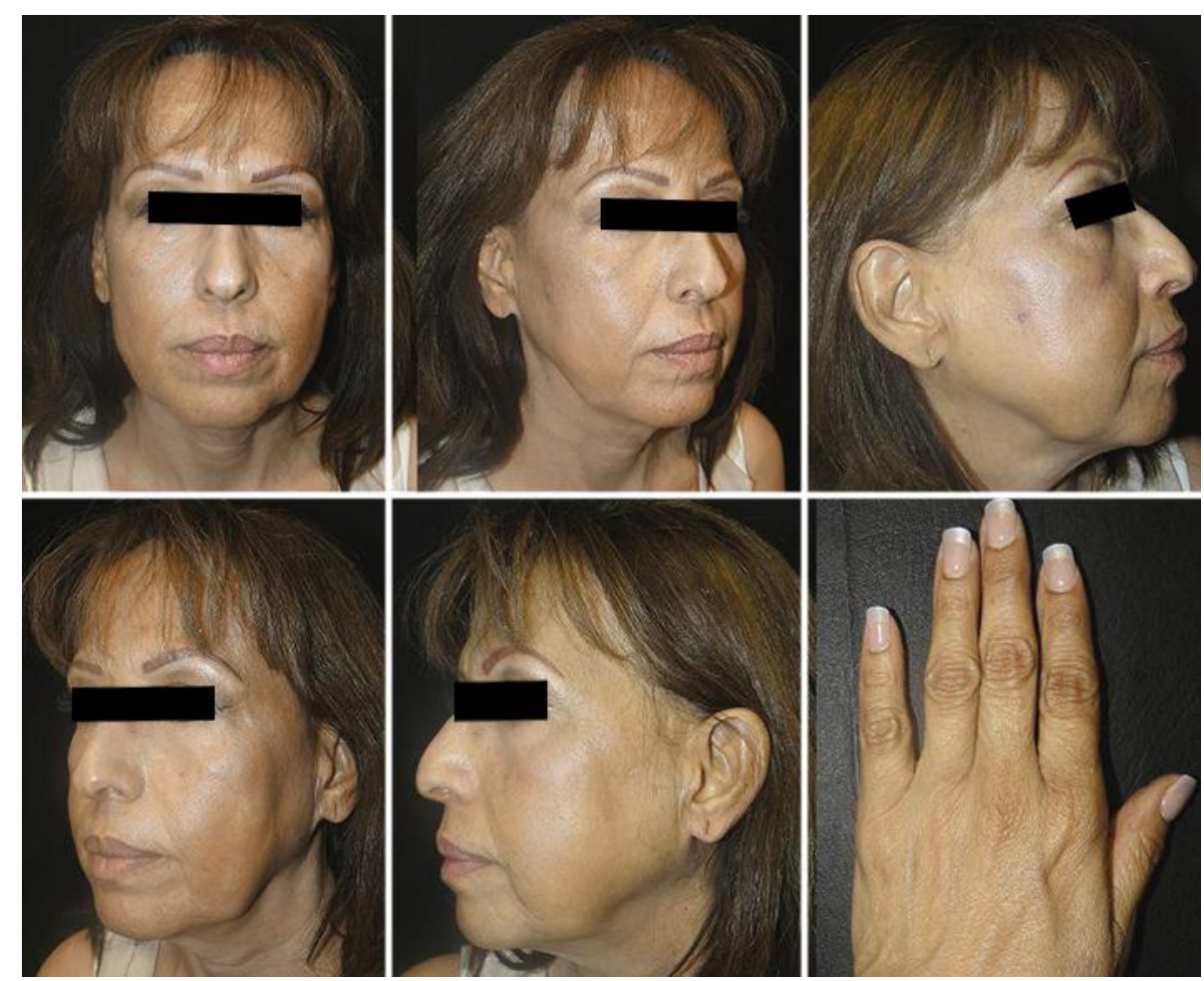

Fig. 3. Five months after treatment suspension, improvement in the lesions can be observed. 